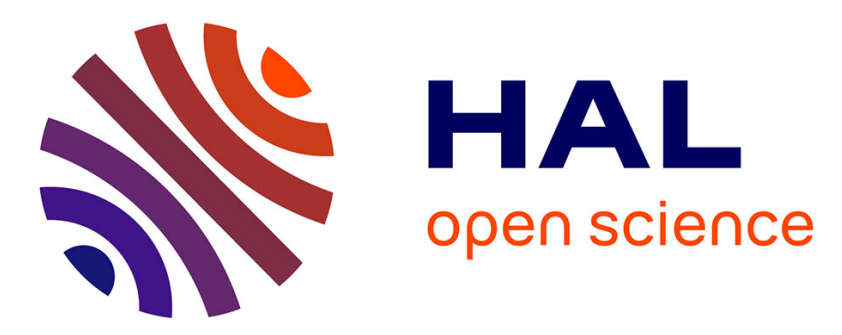

\title{
Shifting gradients of macroscale cortical organization mark the transition from childhood to adolescence
} Hao-Ming Dong, Daniel S Margulies, Xi-Nian Zuo, Avram J Holmes

\section{To cite this version:}

Hao-Ming Dong, Daniel S Margulies, Xi-Nian Zuo, Avram J Holmes. Shifting gradients of macroscale cortical organization mark the transition from childhood to adolescence. Proceedings of the National Academy of Sciences of the United States of America, 2021, 118 (28), pp.e2024448118. 10.1073/pnas.2024448118/-/DCSupplemental . hal-03438638

\section{HAL Id: hal-03438638 \\ https://hal.science/hal-03438638}

Submitted on 21 Nov 2021

HAL is a multi-disciplinary open access archive for the deposit and dissemination of scientific research documents, whether they are published or not. The documents may come from teaching and research institutions in France or abroad, or from public or private research centers.
L'archive ouverte pluridisciplinaire $\mathbf{H A L}$, est destinée au dépôt et à la diffusion de documents scientifiques de niveau recherche, publiés ou non, émanant des établissements d'enseignement et de recherche français ou étrangers, des laboratoires publics ou privés. 


\title{
Shifting gradients of macroscale cortical organization mark the transition from childhood to adolescence
}

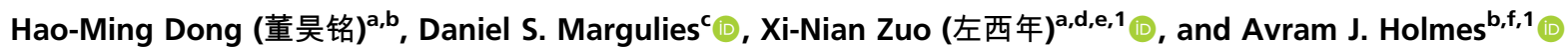 \\ ${ }^{a}$ State Key Laboratory of Cognitive Neuroscience and Learning, International Data Group/McGovern Institute for Brain Research, Beijing Normal University, \\ Beijing 100875, China; 'bepartment of Psychology, Yale University, New Haven, CT 06511; 'CNRS, Integrative Neuroscience and Cognition Center (UMR

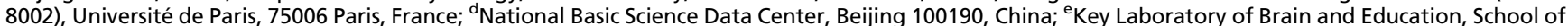 \\ Education Science, Nanning Normal University, Nanning 530001, China; and fDepartment of Psychiatry, Yale University, New Haven, CT 06511
}

Edited by Valerie F. Reyna, Cornell University, Ithaca, NY, and accepted by Editorial Board Member Michael S. Gazzaniga June 1, 2021 (received for review December 3, 2020)

The transition from childhood to adolescence is marked by pronounced shifts in brain structure and function that coincide with the development of physical, cognitive, and social abilities. Prior work in adult populations has characterized the topographical organization of the cortex, revealing macroscale functional gradients that extend from unimodal (somatosensory/motor and visual) regions through the cortical association areas that underpin complex cognition in humans. However, the presence of these core functional gradients across development as well as their maturational course have yet to be established. Here, leveraging 378 resting-state functional MRI scans from 190 healthy individuals aged 6 to $17 \mathrm{y}$ old, we demonstrate that the transition from childhood to adolescence is reflected in the gradual maturation of gradient patterns across the cortical sheet. In children, the overarching organizational gradient is anchored within the unimodal cortex, between somatosensory/motor and visual territories. Conversely, in adolescence, the principal gradient of connectivity transitions into an adult-like spatial framework, with the default network at the opposite end of a spectrum from primary sensory and motor regions. The observed gradient transitions are gradually refined with age, reaching a sharp inflection point in 13 and 14 y olds. Functional maturation was nonuniformly distributed across cortical networks. Unimodal networks reached their mature positions early in development, while association regions, in particular the medial prefrontal cortex, reached a later peak during adolescence. These data reveal age-dependent changes in the macroscale organization of the cortex and suggest the scheduled maturation of functional gradient patterns may be critically important for understanding how cognitive and behavioral capabilities are refined across development.

gradients | cortical organization | development | adolescence | default network

$\mathbf{T}$ he cerebral cortex is comprised of a complex web of large-scale networks that are central to its information processing capabilities $(1,2)$. Although the topographic organization of this distributed network architecture has been characterized in adulthood (3-6), there is a growing consensus that macroscale patterns of functional connectivity are not static across the lifespan (7-12). In particular, the transformative brain changes occurring throughout childhood and adolescence are critical for supporting the emergence and development of physical, cognitive, and social abilities (13-17). Yet, despite clear evidence that patterns of brain organization from neural circuits through large-scale cortical networks relate to behavior (18-20), the age-dependent changes that underpin hierarchical shifts across the functional connectome have yet to be systematically investigated.

Technical advances in the field of connectomics have provided researchers with tools to map the spatial organization of large-scale distributed networks in the human brain $(3,5,6)$. The cortical sheet is comprised of areal units, traditionally defined through their embryological origins, cytoarchitecture, and evoked functions (21-23). Critically, the brain is a multiscale system, and these areal parcels are embedded within segregated processing streams and corresponding networks that are evident through both anatomical projections and patterns of coherent neural activity at rest $(2,24-26)$. This converging evidence suggests the presence of a broad division separating the unimodal somatosensory/motor (somato/motor) and visual territories that form domain-specific hierarchical connections (22) and the heteromodal association areas that integrate long-distance projections from widely distributed sets of brain systems (27). However, the distinction between the unimodal and heteromodal cortex is not reflected in abrupt transitions along the cortical surface. Recent work has revealed that this macroscale property of brain organization is evident in the presence of a principal functional gradient that situates discrete large-scale networks and associated areal parcels along a continuous spectrum, extending from the unimodal systems that underpin perception and action through the association territories implicated in more abstract cognitive functions $(12,28,29)$.

Cortical gradients capture the topography of large-scale networks, offering a complementary approach to standard brain parcellation techniques. Here, rather than marking discrete boundaries at locations of abrupt change in cortical microstructure, function, and/or connectivity, spatial variation across the cortex is examined continuously along overlapping organizing axes $(28,30)$. As such, the gradient approach provides an organizing spatial framework

\section{Significance}

Here, we describe age-dependent shifts in the macroscale organization of cortex in childhood and adolescence. The characterization of functional connectivity patterns in children revealed an overarching organizational framework anchored within the unimodal cortex, between somatosensory/motor and visual regions. Conversely, in adolescents, we observed a transition into an adult-like gradient, situating the default network at the opposite end of a spectrum from primary somatosensory/motor regions. This spatial framework emerged gradually with age, reaching a sharp inflection point at the transition from childhood to adolescence. These data reveal a developmental change from a functional motif first dominated by the distinction between sensory and motor systems and then balanced through interactions with later-maturing aspects of association cortex that support more abstract cognitive functions.

Author contributions: H.-M.D., D.S.M., X.-N.Z., and A.J.H. designed research; H.-M.D. analyzed data; and H.-M.D., D.S.M., X.-N.Z., and A.J.H. wrote the paper.

The authors declare no competing interest.

This article is a PNAS Direct Submission. V.F.R. is a guest editor invited by the Editorial Board.

This open access article is distributed under Creative Commons Attribution-NonCommercialNoDerivatives License 4.0 (CC BY-NC-ND).

${ }^{1}$ To whom correspondence may be addressed. Email: avram.holmes@yale.edu or xinian. zuo@bnu.edu.cn.

This article contains supporting information online at https://www.pnas.org/lookup/suppl/ doi:10.1073/pnas.2024448118/-/DCSupplemental.

Published July 6, 2021. 
for linking multiple large-scale networks and functions in separate domains while avoiding potential biases induced by the inconsistent size and shape of parcels (31). In both humans and nonhuman primates, the association cortex end of the principal gradient is anchored within the default network, including portions of the ventral and dorsal medial prefrontal, posterior/retrosplenial, and inferior parietal cortex (32-34). Converging evidence from tract tracing data in marmosets, for instance, highlights the presence of a gradient of sequential networks radiating outward from the primary cortex to a transmodal network that has many parallels with the human default network (35). The default network acts as a hub that integrates representational information across the cortex $(1,6)$. As such, it constitutes a functional system hypothesized to underpin self-referential processing and core aspects of mental simulation (36). It has recently been proposed that the default network sits at the apex of cortical hierarchy, situated as the most distant association network from the sensory cortex (37). This feature of brain organization may reduce the strong constraints of sensory/motor input on default network function, facilitating the emergence of abstract cognition through the integration of information across modalities.

Suggesting cognitive abilities are closely associated with these macroscale gradients, the spatial framework evident in profiles of intrinsic brain activity mirrors the patterns of functional specialization and flexibility emerging through extrinsic (task-evoked) studies of the human brain (20). These functional gradients have been well characterized in adult populations, providing a framework for describing the integration of local processing streams throughout cortical (28) and subcortical systems (30). The use of low-dimensional representations of functional connectivity provides a unified perspective to efficiently explain core organizing properties of the human cerebral cortex, linking specific regions, networks, and functions. Recent work examining gradients in cortical microstructures has revealed a gradual differentiation of the myelin content of cortical systems from adolescence through young adulthood (38). However, the presence of this overarching organizational structure across development as well as its maturational course have yet to be established.

Human brain development is influenced by a complex series of dynamic processes across biological systems, ranging from shifting profiles of gene expression (39) to hierarchical changes in brain structure and function (14). Although still in its early stages, work in this area has characterized several core organizational principles underlying large-scale network development (40-44). In human and nonhuman primates, for instance, there is an earlier maturational plateau in gray matter (45-47) as well as in synaptic formation and subsequent pruning within unimodal sensory/motor and subcortical territories relative to aspects of the association cortex $(46,48)$. This developmental sequence is reflected in patterns of network activity. For example, brain functions in infancy are characterized by the predominance of short-range connectivity $(49,50)$, which gradually transitions through childhood and adolescence as long-range network connections become increasingly evident $(11,51-56)$. Collectively, these results suggest that brain development may entail a shift from a sensory organization (unimodal gradient) to a globally distributed spatial framework (association gradient) across childhood and adolescence. Wholebrain connectome-wide neurodevelopmental studies have identified patterns of functional network organization that are predictive of behavioral traits (57) and associated with mental health outcomes in adolescence and young adulthood (7). However, to date, there have been few opportunities to directly explore the age-dependent maturation of functional gradients across the cortex. The characterization of age-dependent changes in the macroscale organization of the human brain would provide a tremendous opportunity to understand how connectome development shapes the evolving expression of individual differences in behavior across health and disease.
In examining age-dependent shifts in the macroscale functional organization of the cortex, we applied the dimensionality reduction approach of diffusion map embedding $(28,58)$ to resting-state functional MRI (fMRI) data to extract a global framework that accounts for the dominant connectome-level connectivity patterns in a population of children and adolescents. The resulting components, or gradients, reflect the distillation of complex patterns of local and global functional connectivity across the connectome into simple and interpretable spatial architectures. This approach allowed us to establish the extent to which functional maturation is nonuniformly distributed across cortical networks. In doing so, our analyses revealed the presence of a developmental change from a functional motif first dominated by the distinction between sensory and motor systems and then balanced through interactions with later-maturing connectivity within the association cortex.

\section{Results}

The Dominant Connectivity Gradient Transitions from a Unimodal Network Architecture in Children to Association Cortex in Adolescents. We first decomposed the functional connectivity matrix into components that capture the maximum variance separately for children ( 6 to $12 \mathrm{y}$ of age) and adolescents (12 to $18 \mathrm{y}$ of age). To account for the temporally extended nature of brain development within broad developmental categories (59), follow-up analyses considered participants at 1-y age intervals. Functional connectivity matrices consisted of 20,484 vertices across the cortical sheet derived in data from the Chinese Color Nest Project (CCNP) (60-62). Initial agerelated differences in gradient architecture were evaluated by dividing participants' scans into child $(n=202)$ and adolescent groups $(n=176)$. Consistent with prior work (28), diffusion map embedding (63) was used to decompose participant connectivity matrices, reducing data dimensionality through the nonlinear projection of the vertices into an embedding space. The resulting functional components, more formally termed "manifolds," reflect divergent spatial patterns of connectivity across the cortex, ordered by the variance explained in the initial functional connectivity matrix (SI Appendix, Table 1). Within each component, similar connectivity patterns result in similar values or "connectopies" (29), here referred to as "gradients" (28). A gradient is an axis of variance in cortical features along which areas fall in a spatially continuous order. Areas that resemble each other with respect to the feature of interest occupy similar positions along the gradient. Applied in this manner, diffusion map embedding can distill complex patterns of local and global functional connectivity into simple functional components with interpretable spatial architectures $(28,29,58)$. The resulting gradients are unitless and reflect the position of vertices along an associated embedding axis that captures the primary differences in vertex-level connectivity patterns.

The first two gradients, accounting for the greatest variance in functional connectivity, differed in their spatial organization between children and adolescents (Fig. $1 A$ and SI Appendix, Fig. S1). The resulting gradient architectures were examined relative to prior work in adult populations (28). The significance was established using permuted spin tests, which preserve the spatial autocorrelation structure of effects $(64,65)$. The peak gradient values in children were situated at the central and calcarine sulci, revealing a spectrum differentiating the somato/motor and auditory cortex from the visual system. The first gradient in children closely resembles the second gradient previously identified in adults $\left[p_{\text {spin }}<\right.$ $0.001(28)]$ as well as the second gradient identified in adolescents in the present analyses $\left(p_{\text {spin }}<0.001\right.$; Fig. 2). Conversely, in the adolescent group, the first gradient resembled the principal gradient in adults $\left[p_{\text {spin }}<0.001(28)\right]$. Although one end of the principal gradient of connectivity in adolescents was anchored in the somato/motor and auditory cortex, the regions at the other end encompassed broad swaths of the association cortex, including portions of the ventral and dorsal medial prefrontal, posteromedial/ retrosplenial, and inferior parietal cortex, a spatial pattern that 

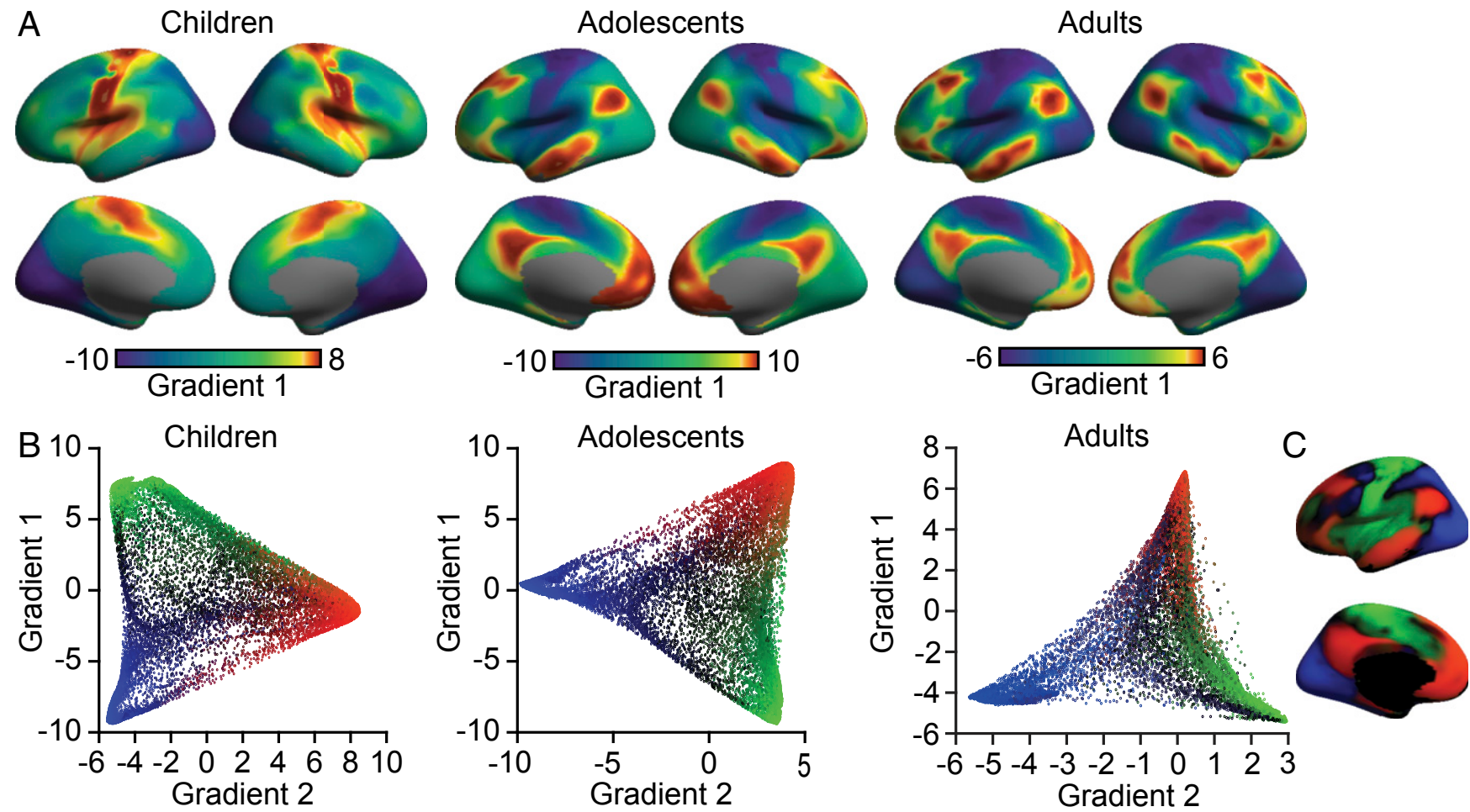

Fig. 1. Functional connectivity gradients differ between childhood and adolescence. (A) The principal gradient of connectivity in children (under 12 y old, Left) peaks within unimodal networks, separating the somato/motor and auditory cortex (red) from the visual cortex (blue). Conversely, in adolescents (12 y old and over, Middle), the principal gradient reveals adult-like organization [Right, data from Margulies et al. (28)], transitioning from the unimodal to association cortex (red). The proximity of colors within each age group indicates the similarity of connectivity patterns across the cortex. Scale bar reflects z-transformed principal gradient values derived from connectivity matrices using diffusion map embedding (59). (B) Scatter plots of the first two connectivity gradients in children, adolescents, and adults. A clear switch within the two gradients accounting for the maximum variance in connectivity is evident between the children and adolescents, with the gradient organization in adolescents transitioning toward the adult form. In children, gradient one separates somato/motor (green) and visual regions (blue), while gradient two distinguishes the unimodal from transmodal cortex (red). These two gradient patterns are flipped in both adolescents and adults. $(C)$ Colors from scatter plot $B$ are presented on of the cortical surface.

corresponds with the canonical default network $(36,37)$. The second gradient in adolescents matched the previously reported second gradient in adults $\left(p_{\text {spin }}<0.001\right)$. Scatter plots further characterize the switch between the two dominant gradient patterns that accompany the transition from childhood through adolescence and into adulthood (Fig. $1 B$ ). Of note, subtle differences in the distribution of head motion, population demographics, and/or data quality can bias estimates of functional connectivity. Therefore, we conducted a set of follow-up analyses rederiving the functional gradients across alternate motion thresholds, incorporating global signal regression, and within sex and motion-matched samples (SI Appendix, Figs. S4-S6). Across each control analysis, the observed effects closely matched the gradient architectures derived through our initial analyses.

The Transition from Childhood to Adolescence Is Marked by Both Dynamic and Stable Functional Gradient Architectures. Previous studies of the macroscale functional organization of the cerebral cortex in adulthood have largely focused on the principal gradient in connectivity in isolation. Having established the presence of distinct gradient topographies in childhood and adolescence, we next examined the consistency of the first six gradients across development (Fig. 2) accounting for the bulk of the functional connectivity variance in children (percent variance: 73.2 ) and adolescents (percent variance: 72.2). To further examine the functional organization of the brain throughout the transition from childhood to adolescence, follow-up analyses rederived the gradient architectures in participants at 1-y age intervals, and associated correlations were calculated (Fig. 2A; reference Dataset 1 for a full set of gradient comparisons by group).

Human brain development is characterized by the early maturation of the unimodal visual and somato/motor cortex, the subsequent refinement of multimodal association areas $(45,66)$, and the gradual incorporation of the visual system into a global processing hierarchy (12). Consistent with these developmental trajectories, the primary gradient of connectivity in children most closely resembled the secondary gradient in adolescents (Pearson's $r=0.80$; $\left.p_{\text {spin }}<0.001\right)$. Furthermore, the secondary gradient of connectivity in children most closely resembled the primary gradient in adolescents $\left(r=0.80 ; p_{\text {spin }}<0.001\right)$.

Permutation analyses examined if the difference in variance accounted for by the first two gradient components in child and adolescent groups (SI Appendix, Fig. S1 and Dataset 1) was greater than a null model in which the group membership is permuted 500 times. Here, the variance accounted for by the unimodal gradient in children (0.38) was greater than in the adolescent group $(0.11$, $P<0.001)$. Conversely, the association cortex anchored gradient accounted for a greater portion of variance in the adolescent $(0.38)$ relative to the child group $(0.12, P<0.001)$. Of note, the secondary gradient in adolescents exhibited a hybrid structure containing both within-unimodal differentiation and a contrast between unimodal and association regions. In adults, the component accounting for the second-most variance in connectivity differentiates regions solely within the unimodal end of the principal gradient (28). While the present analyses are consistent with the initial emergence of an adult-like second gradient in adolescence, future work 
A

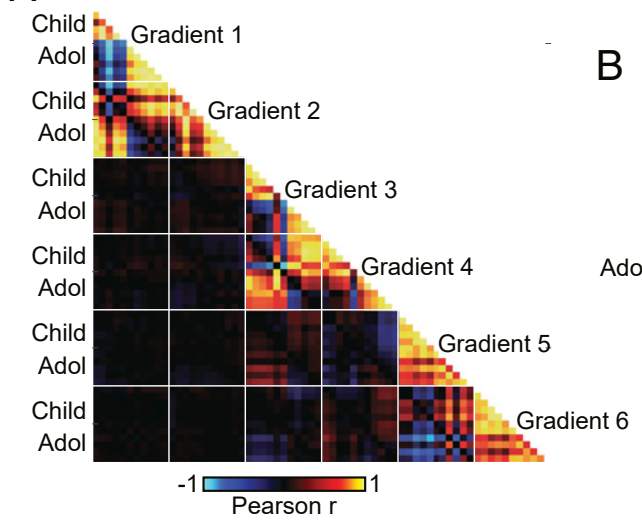

B

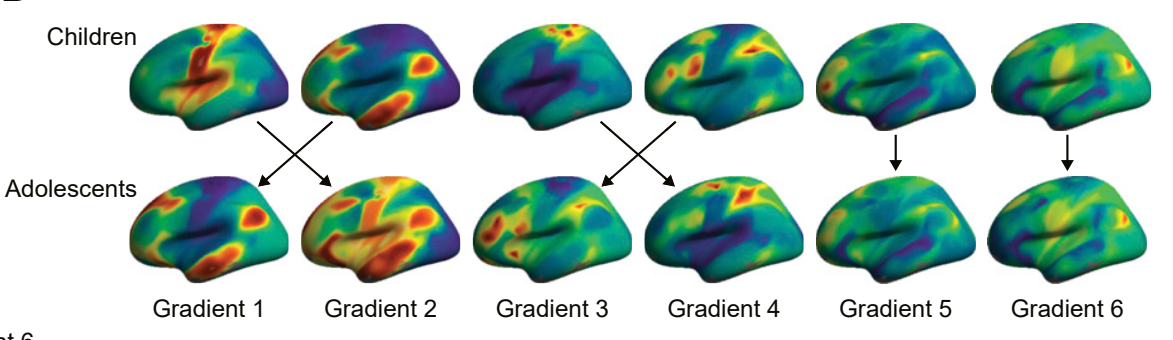

Fig. 2. Evidence for both common and distinct gradient architectures across childhood and adolescence. ( $A$ ) Similarity matrix presents the vertex-level Pearson correlations for the first six gradients across participants grouped at 1-y age intervals. Within-age group correlations for each gradient fall along the center diagonal. Between-age group and cross-gradient correlations are plotted away from the diagonal and reveal both positive (red) and negative (blue) relationships. Participants under 12 y old are labeled as children. Here, associated rows and columns correspond to 6- to 7-, 8-, 9-, 10-, and 11-y-old groups. Adolescent-associated rows and columns correspond to groups of 12-, 13-, 14-, 15-, 16-, and 17-y-old participants. The white lines represent gradient boundaries. $(B)$ Gradient profile maps rendered on cortical surface reveal transitions between the first four gradients across childhood and adolescence. In contrast, the architectures in gradient five and six remained consistent across groups. The black arrows denote maximal vertex-level correlations across age groups for each gradient. Child, children; Adol, adolescents.

should further characterize the trajectories of gradient organization across the lifespan (12).

The developmental transition from a unimodal-centered architecture to transmodal anchored organizing framework was also evident in the third and fourth gradients. Here, the third gradient in children was anchored in the somato/motor cortex, closely resembling the fourth gradient in adolescents $\left(r=0.82 ; p_{\text {spin }}<0.001\right)$. Conversely, the fourth gradient in children, reflecting a network architecture contrasting frontoparietal and somato/motor systems, mirrored the third gradient in adolescents $\left(r=0.85 ; p_{\text {spin }}<0.001\right)$. This pattern of adolescent brain function follows initial reports of gradient topographies in adults (28) and is consistent with a broad developmental transition throughout the cortex to a distributed connectivity structure that encompasses higher-order association areas. The associated age-related refinements in network architecture may facilitate the integration of multimodal information and the segregation of local, specialized processing streams. Importantly, the observed developmental changes were not ubiquitous across all gradients. Highlighting the presence of stable features of the macroscale organization of the cortex, the fifth and sixth gradients were spatially consistent between the child and adolescent groups ( $\left.r \mathrm{~s} \geq 0.90 ; p \mathrm{~s}_{\text {spin }}<0.001\right)$. Moving forward, the analysis of stable and dynamic connectivity structures across development may help determine the unique neurocognitive trajectories that emerge across childhood and adolescence.

Principal Gradients of Connectivity Gradually Transition from a Unimodal to Association Cortex Architecture Across Development. The use of categories, such as child or adolescent, can reveal stable gradient architectures shared within a broad developmental stage. However, many age-specific properties of brain function are lost when central tendencies are examined across large groups, potentially masking subtle shifts associated with the gradual process of neurodevelopment. As one example, the current observation of a hybrid unimodal and association cortex structure for the secondary gradient in adolescents could emerge through at least two potential routes. First, given the age ranges included within the child (6 to $12 \mathrm{y}$ of age) and adolescent (12 to $18 \mathrm{y}$ of age) groups, within-population heterogeneity may obscure or blur the scheduled maturation of functional gradient patterns across broad developmental categories that span many years. An alternate but not mutually exclusive possibility is that adult-like functional organization may emerge later in adolescence, delaying the formation of fully distinct gradient patterns through development. To examine these hypotheses, we rederived the gradient architectures in participants at $1-y$ age intervals.

The principal and secondary gradients of connectivity for each age group are displayed along the lateral surface of the left and right hemispheres in Fig. 3. For the first gradient, participants below $12 \mathrm{y}$ of age displayed unimodal dominant profiles. An abrupt transition was evident in the 12 -y-old participants, and from this point forward, all individuals exhibited an adult-like gradient organization with the default network anchored at the opposite end of a spectrum from primary sensory and motor regions. The second gradient followed an inverse but smoother transition profile across development. Here, the extreme ends of gradient two reflect a mixture of unimodal and association cortices until $\sim 14 \mathrm{y}$ of age. At this point, gradient values within the association cortex gradually decrease, while the contrast between somato/motor and visual regions becomes more apparent. We quantified this trend by extracting the gradient values (standard $z$-scores) within the default network across each 1 -y age interval (Fig. 3B). Across both gradients, age 12 reflected a transition point in which the associated default network gradient values began to reverse. For the first gradient, the relative gradient values within the default network fluctuated around 0 prior to $12 \mathrm{y}$ of age. These data suggest a relatively minimal influence of the default network on this gradient pattern until adolescence. The reversed pattern in the second gradient is consistent with a gradual developmental convergence toward a global network structure in which the association cortex becomes further differentiated, and functional relationships across distinct systems emerge as a dominant feature of cortical organization. Further analysis established that the gradient value within the default network reach a peak later in adolescence (Fig. $3 C$ ). Radar plots reveal the point at which network gradient values peak during development across each of the first six gradients (SI Appendix, Fig. S2).

We next examined the extent to which the first two gradients in childhood and adolescence capture the macroscale layout of canonical large-scale functional networks in adulthood. Here, we examined the Yeo et al. (3) seven-network solution averaged across vertices. Gradient values of all seven functional networks are summarized using box plots in Fig. 4. In line with prior work in adult populations (28), networks were not randomly distributed 


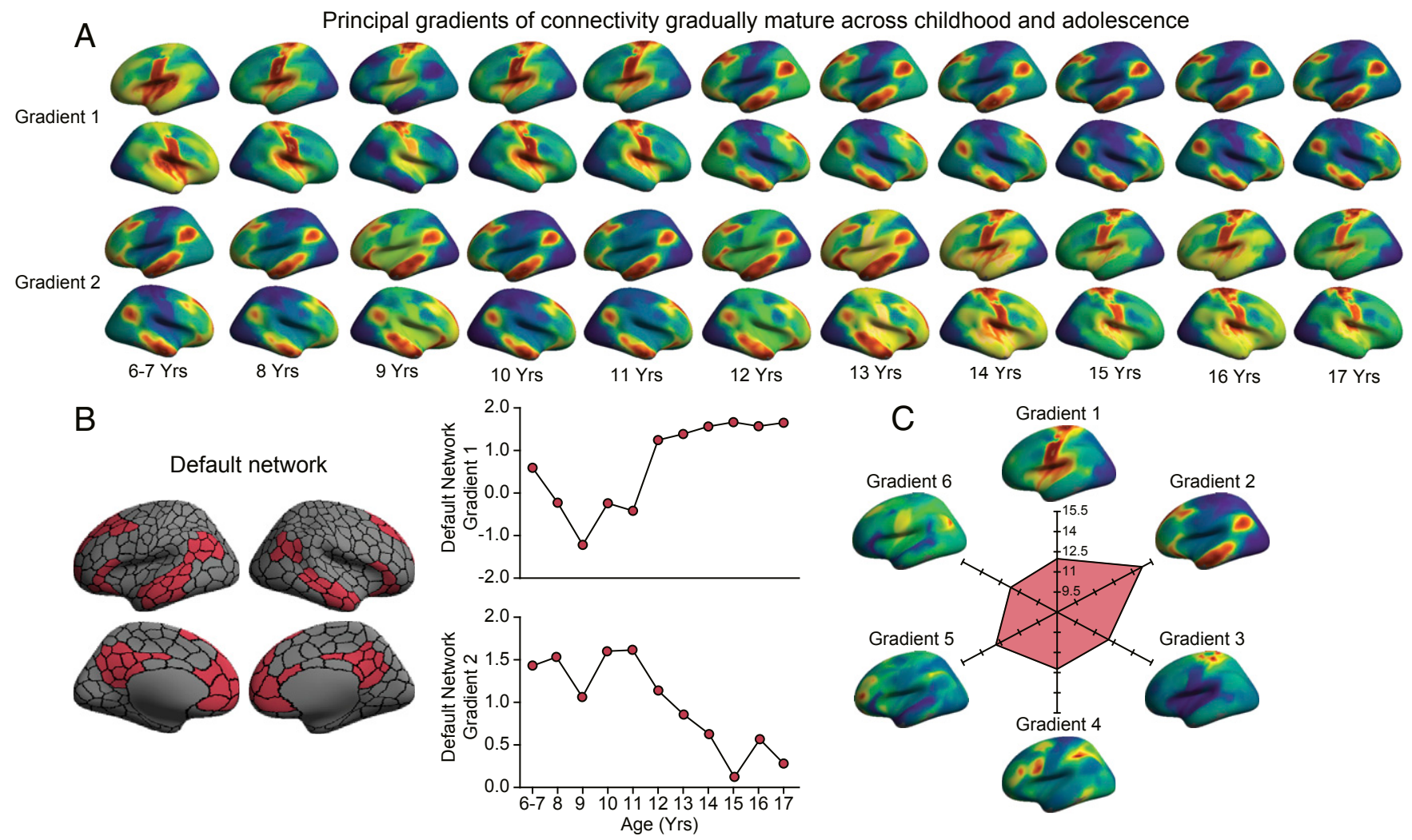

Fig. 3. Functional connectivity gradients gradually mature across childhood and adolescence. (A) Participants are divided into groups at 1-y age intervals. Gradient values are displayed on the lateral surface of the left and right hemispheres in which the proximity of colors within each age group indicates the similarity of connectivity patterns across the cortex. The principal gradient (gradient one), which accounts for the greatest variance in connectivity, segregates unimodal regions in childhood. Across development, a gradual transition was observed in gradient one, reflecting the shift to an adult-like architecture after $12 \mathrm{y}$ of age, at one end anchored by sensory and motor regions and at the other end by the association cortex. Gradient two surface maps reveal the inverse transitional profile across development. Here, the extreme ends of gradient two reflect a mixture of the unimodal and association cortex until $14 \mathrm{y}$ old, at which point gradient values within the association cortex decrease, while the functional separation between the somato/motor and visual regions becomes more prominent. (B) Red parcels denote regions within the default network. The black lines denote parcel and network boundaries based on the Yeo et al. (3) seven-network solution averaged across the 400-parcel functional atlas of Schaefer and colleagues (67). Graphs display average gradient one and gradient two values within the default network for each 1-y bin. (C) Radar plot displays the age at which the default network gradient values peak during development across each of the first six gradients. Radar plots for the remaining large-scale networks are available in SI Appendix, Fig. S2.

along gradients in either children or adolescents. Rather, cortical parcels from the same network tended to cluster at similar positions (Fig. 4B). In children, the somato/motor and visual systems were situated at the extremes along the first gradient. The second gradient in children resembled a transmodal dominant gradient profile, previously reported in adults to follow the spatial constraints of cortical anatomy (28). Here, the default network occupied one extreme position along the second gradient and was maximally separated from the visual system from the perspective of functional connectivity. Conversely, in adolescents, both the first and second gradients consisted of a mixture of unimodal and association cortex architectures. Here, the default network occupied an extreme end of both gradients. Despite this commonality, the first two adolescent gradients were still distinguishable through the distinct relationships of the somato/motor and visual regions. The first gradient of adolescents was characterized by a heteromodal organizational structure in which somato/motor regions were anchored at one end, and the visual system was situated at the center of the spectrum. This contrasts with the second gradient in adolescents in which, although the default network occupied one extreme, somato/motor regions also approached the same gradient terminal with visual regions occupying another end (Fig. 4B). This profile suggests a secondary gradient structure approaching a unimodal-centered organizational scheme but still in progress during adolescence. Collectively, these results demonstrate clear transitions between the principal and secondary gradients of connectivity across childhood and adolescence, providing a framework for the future study of the spatial ordering of large-scale networks during this critical stage for the development of the association cortex.

Functional Maturation Is Nonuniform within Heteromodal and Unimodal Cortex. Our findings detailed above reveal age-dependent changes in the macroscale topographic organization of the cortex, highlighting the transition from a functional motif first dominated by sensory and motor circuits in childhood into one balanced through interactions with the later-maturing association cortex in adolescence. Yet, an important unanswered question remains the extent to which the observed developmental cascades are spatially uniform within large expanses of the cerebral cortex or are nonuniformly distributed at a more granular level both across and within discrete cortical networks.

Here, gradient values were normalized across all surface vertices. Within each gradient map, the age at which the standard gradient value reaches its peak was assigned to each vertex as its "maturation age." The average of the six maturation maps was obtained to represent the process of functional maturation (Fig. $5 A$ ). Critically, functional maturation was not randomly distributed across the cortex; instead, the spatial topography of the maturation map broadly adhered to parcellation borders separating unimodal and 


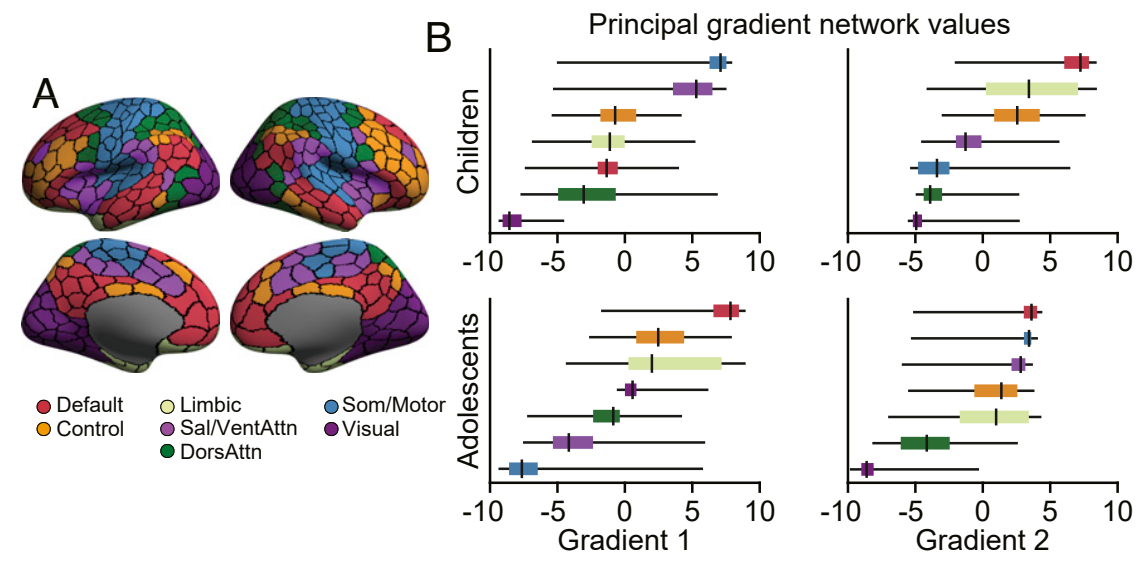

Fig. 4. The default network occupies the extreme end of both principal gradients in adolescence but not childhood. (A) Vertex-level data were summarized based on the Yeo et al. (3) seven networks. (B) The box plots reflect the gradient one and gradient two values averaged within seven networks in children and adolescents ordered by median value. In children, unimodal regions are situated at the extremes along the gradient one, while the default network assumed this position in gradient two. Conversely, in adolescents, the default network occupied the extreme position along the first two dominant gradient patterns.

association cortex $(3,67)$. The visual cortex and somato/motor regions mature earlier in childhood along with portions of anterior insula. The subsequent age at which gradient values peak during development was not uniform within the association cortex. Rather, the early unimodal dominant profile is followed by subsequent gradient peaks within frontoparietal control and attention networks, with limbic and default networks maturing later in adolescence (Fig. 5B). In particular, core default network regions, including the medial prefrontal cortex, parahippocampal gyrus, superior temporal sulcus, and inferior frontal gyrus, were observed to mature latest in adolescence. The maturation map also shows sensitivity in distinguishing functionally heterogenous yet spatially contiguous cortical territories. As one example, the inferior and superior parietal lobules are spatially adjacent, but the former is functionally coupled to the default network, while the latter comprises part of the frontoparietal network. Consistent with the complex functional organization of the parietal lobe, gradient maps revealed tightly interdigitated but differentiated patterns of functional development.

\section{Discussion}

The cerebral cortex is tiled with networks of distinct cortical areas, often delineated by embryology, cytoarchitecture, and evoked functions, broadly linked through continuous gradients of connectivity. In adults, this complex organizational profile can be represented in a low-dimensional space anchored at one end by unimodal regions supporting primary sensory/motor functions and at the other end by the association cortex (28). The presence of this continuous spectrum along the cortical sheet has also been revealed in microstructures, in which regional differences in intracortical myelination emerge gradually through adolescence and young adulthood (38). However, human abilities and behavior change dramatically across development, and the maturational course of these core functional gradients have yet to be established. Here, in a population of school-age children and adolescents, we utilized diffusion map embedding $(28,58)$ to reveal age-dependent shifts in the macroscale organization of the cortex. These analyses demonstrate the presence of a gradual developmental change from a functional connectivity motif in childhood, first dominated by the distinction between unimodal systems and later balanced in adolescence through the maturation of a functional hierarchy that supports more abstract cognitive functions.

Human brain development is marked by a complex series of dynamic processes including hierarchical changes in brain systems that subserve executive functions and affect regulation and social abilities $(14,16,17)$. Of particular relevance for our current analyses, work in animal models has revealed that while synaptogenesis and pruning in the unimodal cortex occurs earlier in life (68), the association cortex is characterized by a protracted period of synaptic development that continues into adolescence $(69,70)$. This staged developmental pattern is consistent with postmortem work in humans, suggesting that by age 12, synaptic pruning within the auditory cortex is complete, a process that extends through midadolescence within the middle frontal gyrus (46). Along the same lines, age-related change is evident in the staggered maturation of cortical gray matter in which the association cortex matures after the unimodal regions (45), at least in part reflecting corresponding shifts in the degree of intracortical myelination (71). The dynamic progression of development from primary sensory and motor systems through networks supporting flexible and abstract cognition likely has a profound impact on the computational landscape of the brain, although studies of
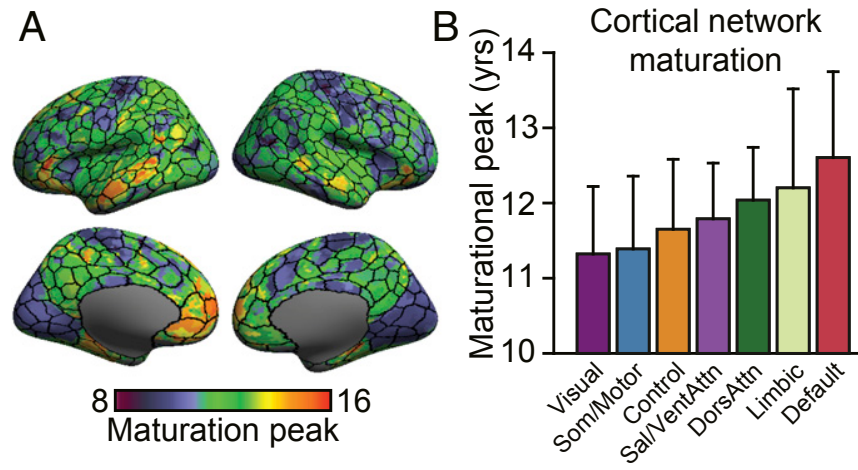

Fig. 5. Functional maturation is nonuniformly distributed across cortical networks. (A) Surface maps display vertex-level functional maturation with the 400-parcel functional atlas [borders from Schaefer and colleagues (67) are overlaid]. Scale bar reflects the ages at which gradient values peak during development. The topography of functional maturation broadly adheres to parcellation borders separating unimodal and association cortex. $(B)$ Age at which the six gradient values peak during development was averaged and plotted within the boundaries of each network. Error bars denote SD. Maturational age broadly follows the cognitive processing hierarchy. Somato/motor and visual networks mature in childhood, while association regions, in particular medial prefrontal aspects of default and limbic networks, peak later during adolescence. 
brain function have often focused on regional approaches or discrete circuits (but see refs. 52, 53, 72, and 73). Here, we extend upon this literature to characterize the macroscale functional organization of the cortex in children and adolescents. Consistent with the development of regional milestones, our results highlight the gradual maturation of broad connectome-level gradients across development, reaching a tipping point around age 13. In children, the bulk of the functional variance was accounted for by a unimodal architecture anchored between somato/motor and visual regions. Conversely, in adolescence, the dominant functional gradient gradually transitioned into an adult-like spatial framework, with the default network at the opposite end of a spectrum from the primary sensory and motor regions. Of note, the current sample of adolescents exhibited less differentiation between transmodal and visual regions, relative to somato/motor. These data suggest the likely continued refinement of the visual system within the global connectivity structure through young adulthood (12).

Although the pattern of cortical maturation was broadly consistent at the network level within unimodal and heteromodal systems, we observed substantial spatial variability in the sequence of gradient development across the cortex. Cortical gray matter develops in a staggered regional manner, with the territories supporting primary functions developing in advance of regions involved in complex and integrative tasks (45). Here, functional maturation broadly followed the cognitive processing hierarchy. Consistent with prior work, somato/motor and visual networks reached a maturational peak in childhood while association regions, in particular temporal and medial prefrontal components of default and limbic networks, peaked later during adolescence. The maturation of the default network typically progresses along a local to distributed pattern. Although the topological profile of the default network has been found as early as the first year after birth (50), local connections are dominant between network hubs during childhood, while distant connectivity is fragmented (53). It is not until late adolescence and early adulthood that dense longrange connections emerge $(9,11)$. In the present analyses, an adult-like default network-anchored gradient was evident during early childhood ( 6 to $12 \mathrm{y}$ of age), accounting for $\sim 10$ to $14 \%$ of the observed functional variance relative to $\sim 34$ to $39 \%$ for the unimodal-centered gradient (SI Appendix, Fig. S1 and Dataset 1). Across development, we observed a gradual transition in the dominant topographic organization of large-scale connectivity, as the amount of variance accounted for by the unimodal spectrum decreased relative to the transmodal dominant gradient architecture. In adolescents (12 to $18 \mathrm{y}$ of age), the adult-like principal gradient accounted for $\sim 33$ to $39 \%$ of the observed functional variance relative to $\sim 10$ to $13 \%$ for the unimodal-centered gradient. These data are consistent with prior regional- and circuit-focused analyses demonstrating the staggered development of the amygdala and the medial prefrontal cortex across childhood and adolescence (14) and associated shifts in response to emotionally evocative stimuli (74-76). Here, the use of diffusion map embedding allowed for the examination of the hierarchical development of multiple networks across childhood and adolescence. Suggesting a broad process of association cortex maturation beyond the canonical default network, age-dependent changes in brain function were not limited to the primary and secondary gradients. For example, across the third and fourth gradients, the increased functional importance of a differentiation of the frontoparietal network became evident during the transition into adolescence.

The present results suggest the age-dependent refinement of a principal gradient that situates discrete large-scale cortical networks along a continuous spectrum, beginning within unimodal inputoutput systems and ending within the default network. These data are consistent with prior theoretical and empirical work examining how brain systems integrate perceptual modalities into deeper layers of cortical processing $(77,78)$, suggesting that cognition should be studied in terms of interdigitated systems and network interactions rather than solely considering discrete/segregated parts in isolation (27). A fundamental question facing the field is the extent to which cortical territories, beyond the primary sensory and motor cortex, possess sharp boundaries across development (see ref. 79). Profiles of gene expression, connectivity, intracortical myelin, and cytoarchitecture provide converging evidence of gradual transitions across the cortical sheet, potentially driving aspects of arealization (80) and suggesting the observed global gradient between sensorimotor and transmodal regions may reflect a key feature of human cortical organization (31). The present data reveal that the process of regional maturation from childhood to adolescence previously observed within circumscribed regions is likely embedded within the development of a principal gradient of macroscale cortical organization. This provides neurodevelopmental details of a generative framework for lifespan development of the human connectome, supporting a gradual shift from a local "anatomically driven" organization to one that is more "topological" and distributed (63). Future studies are needed to better characterize the potential relationships linking observed neurobiological gradients across levels of analyses, their convergence and variation across species, the association with areal and network properties of brain organization, and relations with cognition and behavior throughout the lifespan.

Of note, the reported functional connectivity patterns reflect group averages across subjects. Accordingly, the amount of variance explained within specific networks may be due, at least in part, to associated patterns of intersubject variability, which presents a challenge when working to disentangle the contribution of individual gradient developmental patterns to the observed group gradient architectures (81). Critically, however, the age-dependent transition in gradient architectures allows us to infer the complex reorganization of hierarchical network relationships across development. It has been proposed that the spatial arrangement of areal parcels across the cortical sheet from motor and sensory regions, through attention and cognitive control-related territories, finally terminating at the default network provides anatomical constraints on information processing $(28,37)$. Given the core role of the association cortex-in particular, the default network - in a host of traits and abilities (82), including affect regulation and social functioning (83) as well as increased vulnerability for association cortex-mediated pathologies in adolescence [e.g., affective illnesses (84)], future work should examine possible relationships linking large-scale gradient transitions with cognition, behavior, and symptom burden across development.

The present data are consistent with prior work suggesting a repeating evolutionary and developmental motif embedded within the macroscale properties of human brain organization. Although the fundamental organization of the sensory and motor areas that comprise the unimodal cortex emerged early in vertebrate evolution (85), as brain sizes have increased in primates, the association cortex has disproportionately expanded $(86,87)$. This evolutionary sequence of differential cortical scaling is mirrored across developmental periods as reflected in the staggered embryonic development of the cells that colonize the cortical sheet (88), the formation of subcortical-cortical connections in infancy (8), and the present analyses revealing the gradual refinement of large-scale gradients across childhood and adolescence $(9,53)$. For instance, this is evident in the regulatory role of corticothalamic connections in the formation and maintenance of functional assemblies across the cortex (89). Reflecting the presence of a scheduled maturation in the development of thalamic projections to unimodal and association networks, functional connectivity between the thalamus and sensory/motor cortex is evident in human neonates, while connectivity between the thalamus and association cortex does not emerge until the first year of life (90). Although speculative, such phenomena hint at the possible presence of a universal rule of maturational order in which the same organizing heuristics may be 
invoked multiple times across the lifespan, guiding the development of the cerebral cortex. An important question then arises as to what factors may serve as the early foundation for the protoorganization of the cortex across development and influence subsequent periods of functional refinement during the transition from childhood to adolescence at the onset of puberty.

How the functional architecture of the cerebral cortex forms and is refined throughout development is a central and challenging question across the neurosciences. The present results demonstrate age-dependent changes in the topographic organization of the cortex, transitioning from a gradient separating sensory/ motor modalities into a functional motif, which is later balanced through an increased emphasis on transmodal integration within the association cortex. While a broad process of association cortex maturation was evident across default and frontoparietal networks, we observed substantial spatial variability in developmental timing, indicating that the maturational age of cortical regions was not exclusively driven by spatial proximity. Although our understanding of the functional consequences of gradient refinement across development is incomplete with respect to their roles in behavior across health and disease, the stereotyped progression from unimodal through association cortex architectures nominates candidates for subsequent functional experiments. Whether this phenomenon reflects a universal rule of maturational order in humans remains a hypothesis to be tested in future work.

\section{Methods}

Chinese Color Nest Project. The Chinese Color Nest Project (CCNP) is a 20-y accelerated longitudinal study across the human lifespan (60-62; http:// deepneuro.bnu.edu. $c n / ? p=163$ ). At the baseline of the recruitment, CCNP "growing up in China" invited a total of 198 school-age participants from the Chinese Han population in Chongqing. This accelerated design is particularly valuable for the group-average developmental analysis employed in the present work because each individual was invited to undergo three MRI scans at an interval of 15 mo (balancing season effects), and the longitudinal scans of an individual participant can be included in different age groups. We thus generated a dataset of 176 adolescents and 202 typically developing children. The results are consistent when restricting analyses to a single-scan session for each participant (SI Appendix, Fig. S3). Any participant with a history of neurological or mental disorder, family history of such disorders, organic brain diseases, physical contraindication to MRI scanning, a total Child Behavior Checklist T score higher than 70, or a Wechsler Intelligence Scale for Children intelligence quotient (IQ) standard score lower than 80 were excluded from further analysis (60). All MRI data were obtained with a Siemens Trio 3.0T scanner at the Faculty of Psychology, Southwest University in Chongqing. For each visit, the scanning order was as follows: resting-state fMRI scan (7 $\min 45 \mathrm{~s})$, T1 magnetization prepared - rapid gradient echo (MP-RAGE; $8 \mathrm{~min} 19 \mathrm{~s}$ ), and a resting-state fMRI scan (7 min $45 \mathrm{~s}$ ), giving a total of $15 \mathrm{~min} 30 \mathrm{~s}$ resting-state fMRI scanning. The details regarding participant recruitment and characterization as well as the demographic characteristics of the sample are available in Dong et al. (60). The resting-state scans were acquired with an echo-planar imaging sequence using the following parameters: flip angle $=80^{\circ}$, field of view $=216 \mathrm{~mm}$, matrix $=72 \times 72$, slice thickness $/$ gap $=$ $3.0 / 0.33 \mathrm{~mm}$, repetition time $(T R) / e c h o$ time $=2,500 / 30 \mathrm{~ms}$, slice orientation: sagittal, acquisition direction: interleaved ascending, number of measurements $=184$, and the scanning time lasted for $7 \min 45 \mathrm{~s}$. The reported experiments were approved by the Institutional Review Board from the Institute of Psychology, Chinese Academy of Sciences. All participants and their parents/ guardians gave written informed consent before participating in the study.

MRI Data Preprocessing. Anatomical T1 images were visually inspected to exclude individuals with substantial head motion and structural abnormalities. Next, T1 images were fed into the volBrain pipeline (www.volbrain.upv. es) (91) for noise removal, bias correction, intensity normalization, and brain extraction. All brain extractions underwent visual inspection to ensure tissue integrity. After initial quality checks, T1 images were passed into the Connectome Computation System (CCS; ref. 92) for surface-based analyses. The CCS pipeline is designed for preprocessing multimodal MRI datasets and integrating several publicly available software such as Statistical Parametric Mapping (SPM; ref. 93), FMRIB Software Library (FSL; ref. 94), Analysis of Functional Neurolmages (AFNI; ref. 95), and FreeSurfer (96). For resting-state fMRI data, preprocessing included a series of steps common to intrinsic functional connectivity analyses: 1) dropping the first $10 \mathrm{~s}$ (4 TRs) for the equilibrium of the magnetic field; 2 ) head motion correction; 3 ) slicing timing; 4) de-spiking for the time series; 5 ) estimating head motion parameters; 6) registering functional images to high resolution $\mathrm{T} 1$ images using boundarybased registration; 7) removing nuisance factors such as head motion, cerebrospinal fluid, and white matter signals using ICA-based automatic removal of motion artifacts (ICA-AROMA; ref. 97); 8) removing linear and quadratic trends of the time series; 9) projecting volumetric time series to surface space (the fsaverage 5 model with medial wall masked out); and 10) 6-mm spatial smoothing. All preprocessing scripts are publicly available on github (https:// www.github.com/zuoxinian/CCS). Head motion was estimated using absolute framewise displacement (absFD; ref. 98). Any resting-state scan with a mean absFD above $0.5 \mathrm{~mm}$ was excluded from further analysis. Root mean square FD was also calculated, and the maximum after exclusion was $0.48 \mathrm{~mm}$. The reported results are robust to the inclusion of global signal regression and alternate motion exclusion thresholds (absFD above $0.2 \mathrm{~mm}$; SI Appendix, Fig. S4). The demographic information of subjects included in the analyses is listed in SI Appendix, Table 2. The Pearson correlation between head motion and age in the first resting scan is $r=-0.39, P=2.4 \times 10^{-15}$ and in the second scan is $r=-0.39, P=2.1 \times 10^{-15}$. Increased motion was evident in children (first resting scan: $0.167 \pm 0.096$; second resting scan: $0.092 \pm 0.055$ ) relative to adolescents (first resting scan: $0.108 \pm 0.056$; second resting scan: $0.059 \pm$ 0.031 ; first resting scan: $t=7.13$, degrees of freedom [df] $=376, P=5 \times 10^{-12}$, second resting scan: $t=7.13, \mathrm{df}=376, P=5 \times 10^{-12}$ ). Increased motion was observed in male (first resting scan: $0.160 \pm 0.094$; second resting scan: $0.088 \pm$ 0.053 ) relative to female participants (first resting scan: $0.121 \pm 0.074$; second resting scan: $0.067 \pm 0.041$; first resting scan: $t=4.48, \mathrm{df}=376, P=9.9 \times 10^{-6}$ second resting scan: $t=4.19$, df $\left.=376, P=3.4 \times 10^{-5}\right)$. The results are consistent when restricting analyses to strictly sex (percent female: $50 \%$ ) and motion matching to exclude potential confounding induced by differences in head motion between resting scans. Here, only the second resting scans were used to derive gradients from the child ( $n=162$; second resting scan: absFD $=$ $0.088 \pm 0.052)$ and adolescent groups $(n=122$; second resting scan: absFD $=$ $0.060 \pm 0.027 ; t=-1.69, \mathrm{df}=282, P=0.09 ;$ SI Appendix, Fig. S5). The results are also consistent when examining gradient development in childhood and adolescence separately for male and female participants (SI Appendix, Fig. S6).

Gradient Analysis. Vertex-level functional connectivity (FC) matrices $(20,484 \times$ 20,484 ) were first generated for each resting scan per visit by calculating the Pearson correlation coefficient between any two pair of vertices along the cortical surface, and then the corresponding Fisher z-transformed values were obtained with a hyperbolic tangent function. Then the two test-retest FC Fisher z-matrices within one visit were averaged to increase the signal to noise ratio and reliability for generating individual FCz matrices (81). Individual FCz matrices were next averaged across individuals to form a grouplevel FCz matrix. Only the top $10 \%$ connections of each vertex were retained to keep consistent with previous studies $(3,28)$, and other elements in the matrix were set to 0 to enforce sparsity. These group-level sparse matrices have been released via the Science Data Bank at the National Basic Science Data Center (99). We then calculated the cosine distance between any two rows of the FCz matrix and subtracted from 1 to obtain a symmetrical similarity matrix.

Diffusion map embedding $(28,58)$ was implemented on the similarity matrix to derive gradients (https://www.github.com/NeuroanatomyAndConnectivity/ gradient_analysis). The gradients were ordered by the variance explained (SI Appendix, Fig. S1). To determine the corresponding relationship of the gradients across age groups, we calculated the Pearson correlation coefficient between any two pairs of gradient maps across 1-y interval age groups (Fig. 2A). The gradient maps were first concatenated within gradients across age groups, for example, gradient one maps in each age group were concatenated to form a matrix, and then the gradient matrices were combined to generate the final global gradient matrix. The gradient maps are row vectors in the final matrix and ordered by age, that is, the first 11 rows are the first gradient maps from age 7 to age 17 .

Gradient maps of the children and adolescents group are summarized in Figs. 1 and 2. The first six gradient maps across 1-y interval age groups are listed in Fig. 3. To quantify the gradient transitions across age groups, we extracted the gradient $z$ values within default network regions as summarized through the Yeo et al. (3) seven-network solution averaged across the 400-parcel functional atlas of Schaefer and colleagues (67) (Fig. 3B). To illustrate the gradient-level maturation of the default network, we averaged the maturation values within the boundaries of seven functional networks in six gradient maturation maps and summarized them in radar maps for each network (Fig. 3C and SI Appendix, Fig. S2). In each radar map, the six dimensions refer to the averaged maturation ages for each gradient. Box plots 
in Fig. 4 display the manner in which gradient values are distributed across large-scale brain networks, ordered by the median value within each network.

Maturation Map. Data from the 6- and 7-y-old participants were used to set the gradient orders. First, the six gradient maps were aligned according to their correlation coefficients (Fig. 2B, statistical significance was estimated by spin test) to ensure the gradient maps represented similar functional architectures across age groups. Here, for instance, the gradients reflecting a unimodal architecture (e.g., gradient one in 6- and 7-y-old participants) were grouped together, while gradients exhibiting the differentiation of unimoda and transmodal regions (e.g., gradient two in 6- and 7-y-old participants) were grouped. Then, the maps were normalized by computing the standard $z$-scores across all surface vertices. For each vertex, the age at which the standard $z$ gradient value reaches its peak was assigned to this vertex to be its "maturation age" (Fig. 5A). For instance, for gradient one, the relative $z$-scores within medial visual areas were larger in $8 \mathrm{y}$ olds than that in other age groups, so then 8 was assigned to these vertexes as their maturation age in gradient one. In total, we generated six maturation maps for six gradient maps. The average of these maturation maps was obtained to represent the process of functional maturation. To explore whether this maturation map

1. M. P. van den Heuvel, O. Sporns, Network hubs in the human brain. Trends Cogn. Sci. 17, 683-696 (2013)

2. D. S. Bassett, O. Sporns, Network neuroscience. Nat. Neurosci. 20, 353-364 (2017)

3. B. T. Yeo et al., The organization of the human cerebral cortex estimated by intrinsic functional connectivity. J. Neurophysiol. 106, 1125-1165 (2011).

4. M. F. Glasser et al., A multi-modal parcellation of human cerebral cortex. Nature 536 171-178 (2016)

5. J. D. Power et al., Functional network organization of the human brain. Neuron $\mathbf{7 2}$, 665-678 (2011)

6. X. N. Zuo et al., Network centrality in the human functional connectome. Cereb. Cortex 22, 1862-1875 (2012)

7. T. Kaufmann et al., Delayed stabilization and individualization in connectome de velopment are related to psychiatric disorders. Nat. Neurosci. 20, 513-515 (2017)

8. J. H. Gilmore, R. C. Knickmeyer, W. Gao, Imaging structural and functional brain development in early childhood. Nat. Rev. Neurosci. 19, 123-137 (2018).

9. K. Supekar et al., Development of functional and structural connectivity within the default mode network in young children. Neuroimage 52, 290-301 (2010).

10. L. Q. Uddin, K. Supekar, V. Menon, Typical and atypical development of functional human brain networks: Insights from resting-state FMRI. Front. Syst. Neurosci. 4, 21 (2010).

11. D. A. Fair et al., Functional brain networks develop from a "local to distributed" organization. PLOS Comput. Biol. 5, e1000381 (2009).

12. K. H. Nenning et al., Joint embedding: A scalable alignment to compare individuals in a connectivity space. Neuroimage 222, 117232 (2020).

13. B. J. Casey, S. Getz, A. Galvan, The adolescent brain. Dev. Rev. 28, $62-77$ (2008).

14. B. J. Casey, A. S. Heller, D. G. Gee, A. O. Cohen, Development of the emotional brain. Neurosci. Lett. 693, 29-34 (2019).

15. J. Decety, P. L. Jackson, J. A. Sommerville, T. Chaminade, A. N. Meltzoff, The neural bases of cooperation and competition: An fMRI investigation. Neuroimage 23 744-751 (2004)

16. B. Luna et al., Maturation of widely distributed brain function subserves cognitive development. Neuroimage 13, 786-793 (2001)

17. N. Tottenham, M. A. Sheridan, A review of adversity, the amygdala and the hippocampus: A consideration of developmental timing. Front. Hum. Neurosci. 3, 68 (2010)

18. K. L. Miller et al., Multimodal population brain imaging in the UK Biobank prospective epidemiological study. Nat. Neurosci. 19, 1523-1536 (2016).

19. S. B. Eickhoff et al., Co-activation patterns distinguish cortical modules, their connectivity and functional differentiation. Neuroimage 57, 938-949 (2011).

20. B. T. Yeo et al., Functional specialization and flexibility in human association cortex Cereb. Cortex 25, 3654-3672 (2015)

21. J. H. Kaas, The organization of neocortex in mammals: Implications for theories of brain function. Annu. Rev. Psychol. 38, 129-151 (1987).

22. D. J. Felleman, D. C. Van Essen, Distributed hierarchical processing in the primat cerebral cortex. Cereb. Cortex 1, 1-47 (1991).

23. S. B. Eickhoff, C. Grefkes, Approaches for the integrated analysis of structure, func tion and connectivity of the human brain. Clin. EEG Neurosci. 42, 107-121 (2011)

24. S. E. Petersen, O. Sporns, Brain networks and cognitive architectures. Neuron 88 207-219 (2015)

25. O. Sporns, The future of network neuroscience. Netw. Neurosci. 1, 1-2 (2017).

26. L. Jiang, X. N. Zuo, Regional homogeneity: A multimodal, multiscale neuroimaging marker of the human connectome. Neuroscientist 22, 486-505 (2016).

27. P. S. Goldman-Rakic, Topography of cognition: Parallel distributed networks in primate association cortex. Annu. Rev. Neurosci. 11, 137-156 (1988).

28. D. S. Margulies et al., Situating the default-mode network along a principal gradient of macroscale cortical organization. Proc. Natl. Acad. Sci. U.S.A. 113, 12574-12579 (2016)

29. K. V. Haak, A. F. Marquand, C. F. Beckmann, Connectopic mapping with resting-state fMRI. Neuroimage 170, 83-94 (2018). follows the order of cognitive processing hierarchy, we average the maturation values within boundaries of the seven functional networks (3) as visualized in the Fig. $5 B$ bar graph. The corresponding error bars denote SDs across vertices.

Data Availability. The group-level connectivity matrices are available at Science Data Bank, http://www.doi.org/10.11922/sciencedb.00886 (99). Other CCNP data may be shared upon request. Associated analysis code is available upon request.

ACKNOWLEDGMENTS. This work was supported by the Beijing Municipal Science and Tech Commission (Grants Z161100002616023, Z171100000117012, and Z181100001518003 to X.-N.Z.), the Natural Science Foundation of China (Grant 81220108014 to X.-N.Z.), the Major Project of National Social Science Foundation of China (Grant 20\&ZD296 to X.-N.Z.), the China-Netherlands "Chinese Academy of Sciences-Dutch Research Council" Program (Grant 153111 KYSB20160020 to X.-N.Z.), the Startup Funds for Leading Talents at Beijing Normal University (to X.-N.Z.), the National Basic Science Data Center "Chinese Data-sharing Warehouse for In-vivo Imaging Brain" Program (Grant NBSDC-DB-15 to X.-N.Z.), Key-Area Research and Development Program of Guangdong Province (Grant 2019B030335001 to X.-N.Z.), and Guangxi BaGui Scholarship (Grant 201621 to X.-N.Z.), the European Research Council (Grant 866533 to D.S.M.), and the NIH (Grant R01MH120080 to A.J.H.).

30. Y. Tian, D. S. Margulies, M. Breakspear, A. Zalesky, Topographic organization of the human subcortex unveiled with functional connectivity gradients. Nat. Neurosci. 23, 1421-1432 (2020).

31. J. M. Huntenburg, P. L. Bazin, D. S. Margulies, Large-scale gradients in human cortical organization. Trends Cogn. Sci. 22, 21-31 (2018).

32. D. A. Gusnard, E. Akbudak, G. L. Shulman, M. E. Raichle, Medial prefrontal cortex and self-referential mental activity: Relation to a default mode of brain function. Proc. Natl. Acad. Sci. U.S.A. 98, 4259-4264 (2001).

33. M. E. Raichle et al., A default mode of brain function. Proc. Natl. Acad. Sci. U.S.A. 98 , 676-682 (2001)

34. D. A. Gusnard, M. E. Raichle, Searching for a baseline: Functional imaging and the resting human brain. Nat. Rev. Neurosci. 2, 685-694 (2001).

35. R. L. Buckner, D. S. Margulies, Macroscale cortical organization and a default-like apex transmodal network in the marmoset monkey. Nat. Commun. 10, 1976 (2019).

36. R. L. Buckner, J. R. Andrews-Hanna, D. L. Schacter, The brain's default network: Anatomy, function, and relevance to disease. Ann. N. Y. Acad. Sci. 1124, 1-38 (2008).

37. R. L. Buckner, L. M. DiNicola, The brain's default network: Updated anatomy, physiology and evolving insights. Nat. Rev. Neurosci. 20, 593-608 (2019).

38. C. Paquola et al. and NSPN Consortium, Shifts in myeloarchitecture characterise adolescent development of cortical gradients. eLife 8, e50482 (2019).

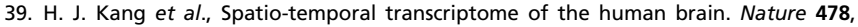
483-489 (2011)

40. G. Collin, M. P. van den Heuvel, The ontogeny of the human connectome: Development and dynamic changes of brain connectivity across the life span. Neuroscientist 19, 616-628 (2013)

41. P. Hagmann, P. E. Grant, D. A. Fair, MR connectomics: A conceptual framework for studying the developing brain. Front. Syst. Neurosci. 6, 43 (2012)

42. V. Menon, Developmental pathways to functional brain networks: Emerging principles. Trends Cogn. Sci. 17, 627-640 (2013)

43. J. D. Power, D. A. Fair, B. L. Schlaggar, S. E. Petersen, The development of human functional brain networks. Neuron 67, 735-748 (2010).

44. A. C. Vogel, J. D. Power, S. E. Petersen, B. L. Schlaggar, Development of the brain's functional network architecture. Neuropsychol. Rev. 20, 362-375 (2010)

45. N. Gogtay et al., Dynamic mapping of human cortical development during childhood through early adulthood. Proc. Natl. Acad. Sci. U.S.A. 101, 8174-8179 (2004).

46. P. R. Huttenlocher, A. S. Dabholkar, Regional differences in synaptogenesis in human cerebral cortex. J. Comp. Neurol. 387, 167-178 (1997).

47. Z. Petanjek et al., Extraordinary neoteny of synaptic spines in the human prefrontal cortex. Proc. Natl. Acad. Sci. U.S.A. 108, 13281-13286 (2011).

48. J. P. Bourgeois, P. S. Goldman-Rakic, P. Rakic, Synaptogenesis in the prefrontal cortex of rhesus monkeys. Cereb. Cortex 4, 78-96 (1994).

49. P. Fransson et al., Resting-state networks in the infant brain. Proc. Natl. Acad. Sci. U.S.A. 104, 15531-15536 (2007).

50. W. Gao et al., Temporal and spatial evolution of brain network topology during the first two years of life. PLoS One 6, e25278 (2011)

51. N. U. Dosenbach et al., Prediction of individual brain maturity using fMRI. Science 329, 1358-1361 (2010).

52. D. A. Fair et al., Development of distinct control networks through segregation and integration. Proc. Natl. Acad. Sci. U.S.A. 104, 13507-13512 (2007)

53. D. A. Fair et al., The maturing architecture of the brain's default network. Proc. Natl. Acad. Sci. U.S.A. 105, 4028-4032 (2008).

54. P. Hagmann et al., White matter maturation reshapes structural connectivity in the late developing human brain. Proc. NatI. Acad. Sci. U.S.A. 107, 19067-19072 (2010)

55. A. M. Kelly et al., Development of anterior cingulate functional connectivity from late childhood to early adulthood. Cereb. Cortex 19, 640-657 (2009).

56. K. Supekar, M. Musen, V. Menon, Development of large-scale functional brain networks in children. PLoS Biol. 7, e1000157 (2009).

57. J. Chen et al., Shared and unique brain network features predict cognition, personality and mental health in childhood. bioRxiv [Preprint] (2020). https://doi.org/10. $1101 / 2020.06 .24 .168724$ (Accessed 20 May 2021). 
58. G. Langs et al., Identifying shared brain networks in individuals by decoupling functional and anatomical variability. Cereb. Cortex 26, 4004-4014 (2016).

59. S. J. Blakemore, S. Burnett, R. E. Dahl, The role of puberty in the developing adolescent brain. Hum. Brain Mapp. 31, 926-933 (2010).

60. H.-M. Dong et al., Charting brain growth in tandem with brain templates at school age. Sci. Bull. 65, 1924-1934 (2020).

61. S. Liu et al., Cohort profile: Chinese Color Nest Project. PsyArXiv [Preprint] (2020). https://doi.org/10.31234/osf.io/d8kpx (Accessed 20 May 2021).

62. X. N. Zuo et al., Human connectomics across the life span. Trends Cogn. Sci. 21, 32-45 (2017).

63. R. R. Coifman, S. Lafon, Diffusion maps. Appl. Comput. Harmon. Anal. 21, 5-30 (2006).

64. E. M. Gordon et al., Generation and evaluation of a cortical area parcellation from resting-state correlations. Cereb. Cortex 26, 288-303 (2016).

65. A. F. Alexander-Bloch et al., On testing for spatial correspondence between maps of human brain structure and function. Neuroimage 178, 540-551 (2018).

66. J. N. Giedd et al., Brain development during childhood and adolescence: A longitudinal MRI study. Nat. Neurosci. 2, 861-863 (1999).

67. A. Schaefer et al., Local-global parcellation of the human cerebral cortex from intrinsic functional connectivity MRI. Cereb. Cortex 28, 3095-3114 (2018).

68. B. G. Cragg, The development of synapses in the visual system of the cat. J. Comp. Neurol. 160, 147-166 (1975).

69. P. R. Huttenlocher, C. De Courten, L. J. Garey, H. Van der Loos, Synaptic development in human cerebral cortex. Int. J. Neurol. 16-17, 144-154 (1982-1983).

70. P. R. Huttenlocher, Synaptic density in human frontal cortex - Developmental changes and effects of aging. Brain Res. 163, 195-205 (1979).

71. J. S. Perrin et al., Growth of white matter in the adolescent brain: Role of testosterone and androgen receptor. J. Neurosci. 28, 9519-9524 (2008).

72. S. Marek, K. Hwang, W. Foran, M. N. Hallquist, B. Luna, The contribution of network organization and integration to the development of cognitive control. PLOS Biol. 13 e1002328 (2015).

73. B. Luna, S. Marek, B. Larsen, B. Tervo-Clemmens, R. Chahal, An integrative model of the maturation of cognitive control. Annu. Rev. Neurosci. 38, 151-170 (2015)

74. D. G. Gee et al., A developmental shift from positive to negative connectivity in human amygdala-prefrontal circuitry. J. Neurosci. 33, 4584-4593 (2013).

75. L. H. Somerville, T. Hare, B. J. Casey, Frontostriatal maturation predicts cognitive control failure to appetitive cues in adolescents. J. Cogn. Neurosci. 23, 2123-2134 (2011).

76. T. A. Hare et al., Biological substrates of emotional reactivity and regulation in adolescence during an emotional go-nogo task. Biol. Psychiatry 63, 927-934 (2008).

77. M. M. Mesulam, From sensation to cognition. Brain 121, 1013-1052 (1998).

78. J. Sepulcre, M. R. Sabuncu, T. B. Yeo, H. Liu, K. A. Johnson, Stepwise connectivity of the modal cortex reveals the multimodal organization of the human brain J. Neurosci. 32, 10649-10661 (2012)

79. F. Krienen, R. Buckner, "Human association cortex: Expanded, untethered, neotenous, and plastic" in Evolutionary Neuroscience, J. H. Kaas, Ed. (Elsevier, 2020), pp. 845-860.
80. D. D. O'Leary, S. J. Chou, S. Sahara, Area patterning of the mammalian cortex. Neuron 56, 252-269 (2007)

81. X. N. Zuo, T. Xu, M. P. Milham, Harnessing reliability for neuroscience research. Nat Hum. Behav. 3, 768-771 (2019)

82. S. M. Smith et al., A positive-negative mode of population covariation links brain connectivity, demographics and behavior. Nat. Neurosci. 18, 1565-1567 (2015).

83. J. R. Andrews-Hanna, J. Smallwood, R. N. Spreng, The default network and selfgenerated thought: Component processes, dynamic control, and clinical relevance. Ann. N. Y. Acad. Sci. 1316, 29-52 (2014)

84. A. Thapar, S. Collishaw, D. S. Pine, A. K. Thapar, Depression in adolescence. Lancet 379, 1056-1067 (2012).

85. S. M. Suryanarayana, J. Pérez-Fernández, B. Robertson, S. Grillner, The evolutionary origin of visual and somatosensory representation in the vertebrate pallium. Nat. Ecol. Evol. 4, 639-651 (2020).

86. R. L. Buckner, F. M. Krienen, The evolution of distributed association networks in the human brain. Trends Cogn. Sci. 17, 648-665 (2013).

87. L. Krubitzer, D. M. Kahn, Nature versus nurture revisited: An old idea with a new twist. Prog. Neurobiol. 70, 33-52 (2003)

88. B. Clancy, R. B. Darlington, B. L. Finlay, Translating developmental time across mammalian species. Neuroscience 105, 7-17 (2001)

89. S. Shipp, The functional logic of cortico-pulvinar connections. Philos. Trans. R. Soc. Lond. B Biol. Sci. 358, 1605-1624 (2003).

90. S. Alcauter et al., Development of thalamocortical connectivity during infancy and its cognitive correlations. J. Neurosci. 34, 9067-9075 (2014).

91. J. V. Manjón, P. Coupé, volBrain: An online MRI brain volumetry system. Front. Neuroinform. 10, 30 (2016).

92. T. Xu, Z. Yang, L. Jiang, X.-X. Xing, X.-N. Zuo, A connectome computation system for discovery science of brain. Sci. Bull. 60, 86-95 (2015).

93. K. J. Friston et al., Statistical parametric maps in functional imaging: A general linear approach. Hum. Brain Mapp. 2, 189-210 (1994).

94. M. Jenkinson, C. F. Beckmann, T. E. Behrens, M. W. Woolrich, S. M. Smith, Fsl. Neuroimage 62, 782-790 (2012)

95. R. W. Cox, AFNI: Software for analysis and visualization of functional magnetic resonance neuroimages. Comput. Biomed. Res. 29, 162-173 (1996).

96. B. Fischl, FreeSurfer. Neuroimage 62, 774-781 (2012).

97. R. H. R. Pruim et al., ICA-AROMA: A robust ICA-based strategy for removing motion artifacts from fMRI data. Neuroimage 112, 267-277 (2015)

98. J. D. Power, K. A. Barnes, A. Z. Snyder, B. L. Schlaggar, S. E. Petersen, Spurious but systematic correlations in functional connectivity MRI networks arise from subject motion. Neuroimage 59, 2142-2154 (2012).

99. H. M. Dong, X. N. Zuo, Age-specific brain funtional connectivity matrices for children and adolescents at school age. Sci. Data Bank, 10.11922/sciencedb.00886 (2021) 